\title{
Les avatars de l'autorité sur Twitter : l'exemple des usagers face à l'Allocution de Nouvel An du Président Macron
}

Authority and Twitter: Users facing President Macron's New Year's Eve Message

\author{
Keren Sadoun-Kerber
}

\section{(2) OpenEdition Journals}

\section{Electronic version}

URL: http://journals.openedition.org/aad/5034

DOI: 10.4000/aad.5034

ISSN: 1565-8961

\section{Publisher}

Université de Tel-Aviv

\section{Electronic reference}

Keren Sadoun-Kerber, "Les avatars de l'autorité sur Twitter : l'exemple des usagers face à l'Allocution de Nouvel An du Président Macron", Argumentation et Analyse du Discours [Online], 26 | 2021, Online since 14 April 2021, connection on 16 April 2021. URL: http://journals.openedition.org/aad/5034 ; DOI: https://doi.org/10.4000/aad.5034

This text was automatically generated on 16 April 2021.

\section{(†)

Argumentation \& analyse du discours est mis à disposition selon les termes de la licence Creative Commons Attribution - Pas d'Utilisation Commerciale - Pas de Modification 4.0 International. 


\title{
Les avatars de l'autorité sur Twitter : l'exemple des usagers face à l'Allocution de Nouvel An du Président Macron
}

\author{
Authority and Twitter: Users facing President Macron's New Year's Eve Message
}

Keren Sadoun-Kerber

\section{Introduction}

1 L'autorité politique semble aujourd'hui particulièrement contestée. Son ébranlement se traduit par un éparpillement de ses sources impersonnelles (la religion, la tradition) aussi bien que personnelles : elles ne sont plus partagées par tous, si bien qu'il ne s'agit plus, comme le disait Hannah Arendt (1972 [1954] : 121) de "prendre appui sur des expériences authentiques et indiscutables, communes à tous ». Dans la sphère politique, l'affaiblissement de l'autorité qui touche tous les domaines est encore plus saillante en raison de la méfiance grandissante du public à l'égard de ses dirigeants ; la confiance du public dans les élus est désormais ébranlée (Revault d'Allonnes 2012).

Depuis le constat d'Arendt, nous avons été témoins de la montée des réseaux sociaux, qui occupent de nos jours une place croissante dans notre vie quotidienne. Or, cette plateforme sociale se veut égalitaire (Candel et Gkouskou-Giannakou 2017); les hiérarchies traditionnelles entre dirigeants et citoyens y sont en apparence abolies : les citoyens peuvent réagir " directement » à la parole présidentielle, et ils sont invités à participer à l'activité citoyenne (Longhi 2019). L'importance de ces réseaux dérive de leur omniprésence dans notre existence. Nous y sommes exposés même lorsque nous ne les utilisons pas directement. Ainsi, par exemple, les tweets qui constituent un genre de discours politique (Paveau 2006, 2013, Longhi 2013), sont rapportés dans les médias " traditionnels ». Il en va de même dans le sens inverse : les discours traditionnels sont souvent affichés sur les réseaux sociaux et nous suivent jusqu'à nos choix paramétrés. 
La présence de ces réseaux facilite par ailleurs le groupement d'individus et l'incitation à la manifestation, comme dans le cas récent de la montée du mouvement des Gilets jaunes (Shebach et al. 2018), et bien d'autres encore. Ces réseaux sont donc un terreau fertile pour un regroupement de personnes qui souvent ne se connaissaient pas, à des fins de discussion, voire d'action collective.

Cet article propose une analyse de la réception de l'autorité d'un dirigeant politique sur Twitter. Il s'agit d'examiner des réactions en ligne à une autorité de type institutionnel. Le cas de figure analysé est celui d'Emmanuel Macron lors de son allocution de Nouvel An en $2018^{1}$. L'objectif est de mieux comprendre à travers cette analyse le phénomène de la mise en question de l'autorité du politique sur les réseaux sociaux.

Selon quelles modalités Twitter permet-il aux usagers d'attaquer, voire de rejeter l'autorité du Président de la République, et dans quelle mesure ses caractéristiques favorisent-elles une telle remise en cause? S'agit-il d'un rejet de l'autorité présidentielle en soi, ou de l'autorité spécifique de Macron, qu'on souhaiterait remplacer par une autre? S'agit-il d'un rejet argumenté et rationnel, ou purement émotionnel et dépourvu de rationalité ? Y-a-t-il là des propositions concrètes ou seulement un besoin de catharsis, sinon de défoulement ? Par ailleurs, on examinera de quels procédés usent les utilisateurs de Twitter qui s'efforcent au contraire de renforcer l'autorité contestée de Macron. Enfin, on se demandera s'il y a une vraie "conversation» entre les internautes, et à quel point les réactions sont dichotomiques et attisent la polémique (Amossy 2014). À travers ce questionnement, nous aspirons à mieux comprendre la singularité du rapport à l'autorité sur Twitter.

Pour ce faire, nous analysons des réactions des usagers à l'allocution présidentielle au prisme du critère de l'autorité : celles qui la renforcent, et celles qui la contestent. Le corpus contient uniquement les réactions à @EmmanuelMacron et non pas tous les 3500 retweets avec ou sans commentaires. La raison en est le besoin pratique de limiter un corpus énorme, ainsi que de se focaliser sur la réaction au discours de Macron de la façon la plus «directe». Cependant, nous allons nous attarder un moment sur deux enchaînements spécifiques de réactions afin d'en saisir la dynamique.

\section{De la plateforme Twitter}

6 Selon la page affichant les règles de Twitter, l'objectif de cette plateforme est « de servir la conversation ${ }^{2}$ publique» (je traduis de l'anglais). Effectivement, "les écrits numériques sont fondamentalement des écrits conversationnels » (Marcoccia 2016 :11). Le trait de l'égalité qui caractérise la conversation se joint à une sorte de « démocratisation » de l'expression des citoyens, caractéristique de ces réseaux (Candel et Gkouskou-Giannakou $2017: 7$ ). Tout un chacun peut s'exprimer sur la toile, et chaque opinion est censée être « légitime », au nom d'une conception libérale et pluraliste qui règne dans le monde occidental. De plus, chaque opinion est souvent mise à égalité avec d'autres, de façon à brouiller davantage encore les hiérarchies.

7 Mentionnons d'autres caractéristiques de Twitter pertinentes pour notre sujet, qui conditionnent le débat qui s'y déroule : (1) L'anonymat (Herring 20073) : la possibilité de masquer son identité offre d'autres manières de débattre qu'à visage découvert, pour le meilleur ou pour le pire - ils tendent à être plus violents verbalement (Marcoccia 2016 : 32). (2) Les normes : il existe des règles de comportement acceptées, ce que l'on appelle nétiquette (Herring 2007) ou Twittétiquette (Paveau 2006, 2013). Néanmoins, il y a une 
grande latitude de possibilités d'expression permettant des zones grises par rapport à ces règles (interdictions d'affichage de diffamation, de racisme, etc.), au-delà desquelles les tweets peuvent être effacés. (3) Le cadre participatif: le caractère visible des échanges. (4) Les participants : ils varient selon chaque paramètre (âge, sexe, etc.) et surtout selon l'idéologie à laquelle ils adhèrent, ce qui peut provoquer des polémiques. (5) Le ton : les messages sur Twitter peuvent véhiculer une tonalité à l'aide de différents moyens. (6) La taille: le message posté est limité à 280 signes, ce qui peut réduire le développement d'une pensée, en exigeant d'en afficher la quintessence (bien qu'il y ait des moyens de contourner la contrainte). Cette caractéristique peut encourager la production de "phrase choc", ou de "formules" avec pour objectif d'obtenir un message plus percutant (Longhi 2013), donc digne d'être « liké » ou « retweeté ». (7) La plurisémioticité : cette plateforme se caractérise par une multitude de systèmes de signes (Marcoccia 2016), visuels, auditifs et verbaux. Parmi les possibilités, il y a le mème et le gif (mème animé) qui constituent des éléments visuels et culturels (Dawkins 1976), souvent une blague, transmissible en ligne et malléable (Davison 2009). Ces unités culturelles et mimétiques sont « la raison d'être de la communication digitale ", selon Limor Shifman (2013: 373) dans le sens où si l'utilisateur ne les diffuse pas, elles n'existent pas (selon l'expression de Henry Jenkins). Elles peuvent être comprises comme des actes de discours à caractère pluri-sémiotique (image référence culturelle + message écrit) (Wagener 2020), au travers desquelles sont véhiculés des arguments repérables. Enfin, le réseau présente d'autres affordances particulières, une sorte de «twittécriture " (Paveau 2013) incluant des marques délinéarisées : les possibilités de réponse à un utilisateur (@), la présence d'une chaine de réactions à un sujet déterminé par un hashtag (\#), l'option de retweeter (RT) et de fournir des liens.

Deux sortes de discours politiques émanent de cette plateforme : le discours politique qui vient d'en haut - le discours politique classique - et le discours politique qui vient d'en bas, des citoyens. Dans le cas présent, il s'agit d'un discours traditionnel qui est diffusé à la télévision, et non pas d'un tweet où l'homme politique formule un énoncé bref. Dans le discours médié (mediated discourse), dont Twitter fait partie, les utilisateurs de Twitter ${ }^{4}$ ne peuvent interagir directement avec le discours qui vient d'en haut que si l'homme politique le désire. Sans cela, pas d'interaction. Dans le cas précis du Président Macron, il est fort probable que c'est son équipe (community manager) qui gère les messages postés sur le réseau, et non lui-même ${ }^{5}$, en une sorte de mise en scène sur Twitter (Longhi 2013) de la parole présidentielle. Dans ce cas, les internautes peuvent seulement commenter, et entrer en discussion avec d'autres utilisateurs. Ainsi, si le caractère spontané de la communication sur Twitter (ibid.) est perceptible dans les réactions d'utilisateurs lambda, il est rarement réel dans la communication présidentielle, qui est au contraire très contrôlée et réfléchie. L'intérêt d'analyser les réactions au discours réside dans le besoin de vérifier l'écart possible entre l'acte perlocutoire de l'allocution et son effet perlocutoire (Austin 1970).

\section{Cadre conceptuel}

Passons d'abord brièvement en revue quelques théories développées dans le domaine de l'analyse du discours et de l'argumentation qui traitent de l'autorité. La catégorie de l'autorisation, l'une des quatre catégories développées par Van Leeuwen (2007) comme stratégies de légitimation discursives, est ici particulièrement pertinente. Elle 
comporte six sous-catégories : (1) l'autorité personnelle est celle qui est conférée à une personne en raison de son statut ou de sa fonction dans une institution. L'on peut rajouter à cette catégorie la notion d'autorité institutionnelle telle que développée par Bourdieu (1991), qui accorde une importance primordiale au fait que celui qui parle doit être la personne légitime pour le faire, dans une situation légitime et utilisant un langage légitime (ibid. : 109) ; (2) l'autorité de l'expert, doté d'un savoir qui confère une légitimité aux propos. Le fait qu'il existe plusieurs solutions différentes à chaque problème entraîne cependant graduellement une atteinte à l'autorité de l'expert ${ }^{6}$; (3) l'autorité du modèle à suivre. Elle rejoint, comme les catégories (1) et (2), l'argument d'autorité dont parlent Perelman et Olbrechts-Tyteca (1989 [1958]). Cependant, tandis que ces derniers désignent des figures de modèle hiérarchiquement supérieurs (les anciens, les philosophes, etc.), Van Leeuwen met l'accent sur un modèle de dirigeant d'opinion (opinion leader), qui se réfère plutôt à des célébrités, voire des personnes qui appartiennent au même groupe ; (4) l'autorité impersonnelle : les lois, les règles, la politique ; (5) l'autorité de la tradition. Celle-ci est tout particulièrement pertinente pour l'allocution de Nouvel An qui est une tradition (politique). Elle peut se manifester par des vocables comme « habitudes ", « tradition » (" c'est comme cela que nous avons toujours fait »); (6) l'autorité du conformisme: «c'est comme cela que tout le monde fait ».

10 L'autorité se manifeste par le recours à des figures ainsi qu'à des arguments d'autorité. Ceux-ci sont aussi repérables dans le discours qui les met en mots et les mobilise pour ses objectifs propres. En effet, le locuteur cherche à susciter un "surcroit de crédibilité " non seulement à l'aide de son statut social mais aussi par les dimensions discursive et contextuelle du discours (Oger et Monte, 2015). L'autorité dans le discours peut être construite à l'aide de divers éléments. Un phénomène abordé par KriegPlanque (2015) est celui du figement discursif, qui se manifeste dans des formules, slogans et "petites phrases». Ces éléments peuvent être basés sur des croyances collectives convenues, censées être considérés comme allant de soi. Une autre manière de construire le discours d'autorité est d'user d'une phraséologie en émettant par exemple des aphorismes, qui donnent l'impression d'émettre des vérités absolues. Cette impression est construite également par un procédé d'effacement énonciatif (Rabatel 2004) et l'utilisation d'un sujet impersonnel («Il importe de... »).

\section{L'allocution de Nouvel An}

11 En cette année 2018, l'allocution de Nouvel An a été prononcée dans un climat extrêmement tendu, dû aux manifestations des Gilets jaunes qui ont commencé un mois et demi plus tôt (le 17.11.18). Ce mouvement a mis à mal l'image d'Emmanuel Macron (Sebbah et al. 2018), à la différence de l'image qu'il avait projetée lors de sa campagne électorale pendant laquelle il avait fait preuve d'une capacité communicationnelle exceptionnelle (Sadoun-Kerber 2018). A cela s'ajoute la colère suscitée par les différentes réformes controversées, ainsi que l'affaire Benalla, qui a dégradé considérablement la crédibilité du Président.

L'allocution de Nouvel An constitue une tradition de la Cinquième République, comme l'affirme Macron dès l'abord: "Fidèle à une tradition qui nous est chère, je suis heureux de vous présenter tous mes vœux [...]». Ce genre relève de l'exercice du pouvoir par excellence, et est une manifestation de l'autorité institutionnelle. Il s'agit 
en partie de l'autorité qui dérive de la position institutionnelle de l'orateur, dans le sens de Bourdieu (1991) ; cependant, le discours se doit de la renforcer.

On a affaire à un genre épidictique (Leblanc 2016 : 27), dont l'une des caractéristiques est de réunir l'auditoire autour de valeurs partagées. Une autre caractéristique en est l'énumération des réalisations de l'année. Il s'agit d'un bilan politique (ibid.: 43) qui s'ajoute à l'aspiration politique de gérer l'opinion à travers ce discours. Dans cette perspective, l'allocution de Nouvel An contient aussi des éléments du genre délibératif. En effet, dans ce contexte politique précis, il semble que le Président Macron profite de la situation pour faire passer un message (travailler plus, renforcer l'Europe, et d'autres messages d'inspiration néo-libérale). Message qui ne fait pas l'unanimité, comme on peut l'imaginer. Si le discours institutionnel a tendance à effacer les marques de dissensus (Oger et Monte 2015), ce n'est pas tout à fait le cas dans le discours d'Emmanuel Macron.

Bien qu'il y ait un certain nombre de thématiques qui reviennent (la politique intérieure / extérieure, les relations avec l'Europe), chaque Président apporte son ethos personnel, entre autres à travers un choix personnel de vocables, de verbes, de temps, etc. (ibid.) L'un des ethè que l'allocution présidentielle cherche à construire est celui du guide de la France (Mayaffre 2012). Macron le construit discursivement à l'aide d'un certain maniement de la langue : phrases impersonnelles ${ }^{7}$, choix de verbes ${ }^{8}$ manifestant une capacité et une réactivité qui se traduit en leadership. C'est par rapport à ces images d'autorité, ainsi que par rapport aux thèses politiques présentées au public, que les membres de l'auditoire peuvent faire entendre une contestation. Car il y a dans l'allocution Présidentielle à la fois de l'épidictique et la promotion d'un agenda politique précis.

Le support de ce genre de discours est la télévision, les médias traditionnels. Son affichage sur la plateforme de Twitter est une hybridation de supports. L'affichage sur le réseau rend visible l'acceptation ou la contestation du discours et de ses messages dans un climat de protestation continue.

\section{Les résultats des réactions}

Plus de 3.500 réactions et retweets ont été enregistrées sur Twitter, parmi lesquels 519 avec commentaires d'autres internautes, et 3.043 sans commentaires. Les réactions que nous avons analysées sont celles qui se rapportent directement à Macron par la mention @EmmanuelMacron (194), et qui manifestent une tentative d'interaction (unilatérale) avec le Président. Nous avons repéré : (1) 67 réactions renforçant l'autorité de Macron, (2) 123 réactions qui la contestent, (3) quatre réactions qui sont à peu près neutres.

Les tweets contestataires ont été regroupés selon deux types d'argument en fonction de ce qui émane des réactions : argument ad personam (88) qui visent à disqualifier la personne de l'adversaire (Perelman et Olbrechts-Tyteca, 1958:150) ${ }^{9}$ et argument ad hominem (10) visant à réfuter une thèse, bien que sa conséquence soit de toute façon la diminution du prestige de la personne (ibid.). La distinction entre les deux a pour objectif de voir si les réactions visent seulement à disqualifier Emmanuel Macron ou justifient aussi ce discrédit. Parmi les 124 réactions de protestation, il y a 23 réactions qui mélangent les deux. 


\section{Discussion}

Ce qui émane de l'analyse des réactions contestataires est que c'est l'autorité personnelle de Macron qui est mise en question, et non pas l'autorité en tant que telle. Ces réactions de protestation sont exprimées par une majorité d'ad personam mais aussi d'un certain nombre d'ad hominem, dont on discutera à présent la signification. Quant à l'autorité institutionnelle, elle est perçue différemment selon les internautes. Pour certains, les insultes portent uniquement atteinte à l'autorité personnelle tandis que pour d'autres, elles dégradent la fonction et le statut.

La majorité d'attaques ad personam n'est pas surprenante, compte tenu de l'une des caractéristiques du réseau, celle de l'anonymat des internautes (Marcoccia 2016). Ces attaques sont assez agressives et violentes. Pour la plupart, il n'y a aucune tentative de légitimer cette violence, comme s'il n'en était pas besoin. Par contre, ce qui est intéressant, c'est qu'il y a une vingtaine de réactions qui mélangent les deux types d'argument. Ce qu'on peut en tirer comme enseignement est le besoin de certains internautes de légitimer leur réactions violentes. À leurs yeux, il semble qu'une réaction qui s'appuie sur une argumentation plus développée et rationnelle (de type ad hominem qui expose les contradictions entre parole et actes, ou paroles et réalité) puisse être plus convaincante.

Les attaques ad personam s'expriment principalement de deux façons: 1) par des qualifications péjoratives qui visent un trait de personnalité. 2) Par des insultes, voire des grossièretés. Les qualifications visent à disqualifier et dénoncer un certain trait de caractère indésirable. C'est le cas de «menteur ». Ce sont des réactions extrêmes, utilisant des stratégies liées à la polémique, comme la dichotomisation (Amossy 2014). Un grand nombre de réactions affichent l'image du Président comme menteur en l'exprimant en toute lettres sans passer par quatre chemins. L'une d'entre elles provient de @helmut_foster qui affiche un gif de Pinocchio, la fameuse marionnette dont le nez s'allonge lorsqu'elle ment. 


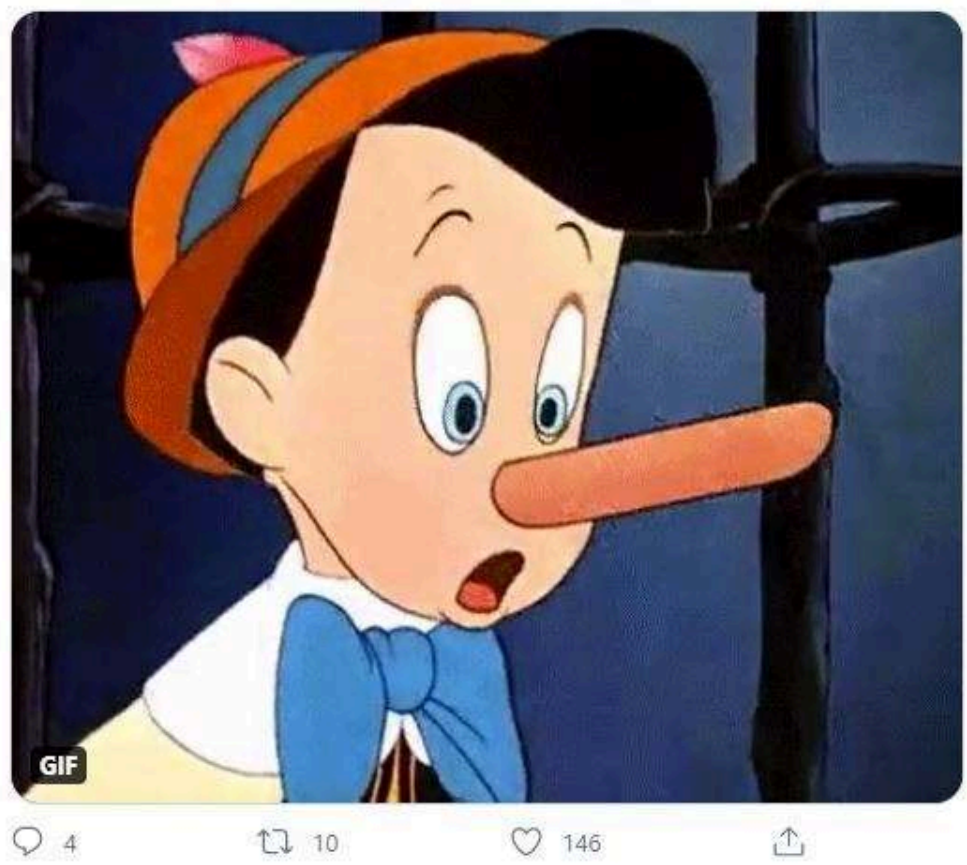

Dans une autre, on voit Macron au nez allongé :

Pinocchio s'est exprimé debout ne lâchant rien ...

Tout va bien semble-t'il nous faire croire, un petit cours d' histoire abrégé et ça passe ....incroyable mais vrai !

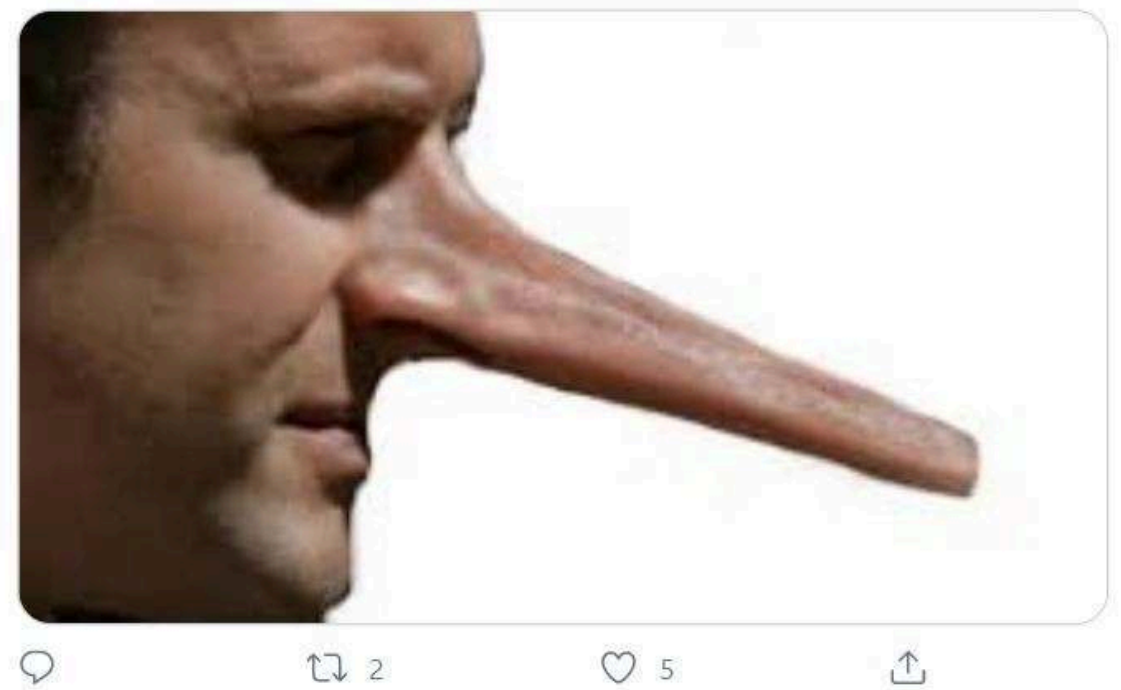

L'utilisation du support visuel de Pinocchio est courante chez les éditorialistes, comme le montre Leo Groarke (2017) qui le dénomme un "argument Pinocchio ». Selon lui, l'utilisation du cartoon (pour nous ici, il s'agit d'un mème) «peut consister en une attaque dévastatrice sur la crédibilité (leur ethos) d'une personne, qui dégrade leur position morale et politique" (ibid.: 106; je traduis de l'anglais). Dans ce cas, l'utilisateur affiche son attitude critique face aux propos et aux idées du Président. Cela 
rejoint la troisième dimension du modèle tripartite (contenu, forme, attitude) du mème (Shifman 2013), celle d'attitude affichée par l'utilisateur du mème. On voit alors que le mème peut devenir un acte de discours (Wagener 2020). La mention de "menteur" peut être considérée comme un cas d'ad personam lorsqu'elle n'est pas soutenue par un argumentaire dévoilant une contradiction entre paroles et actes, ou entre paroles et réalité. Plus loin, nous verrons un autre cas qui est de l'ordre de l'ad hominem (qui constitue une critique plus acerbe que la simple attaque contenue dans l'emploi de «menteur » et qui risque d'affaiblir davantage son autorité que l'insulte).

Les invectives et les grossièretés («con », «idiotie», «connard»), en revanche, ne s'adjoignent pas toujours un argument qui les justifie. Elles expriment une méfiance vis-à-vis de Macron en personne. Mais sont-elles en soi capables d'affaiblir l'autorité du Président? L'une des fonctions de ces grossièretés est celle de défoulement, de catharsis. Le juron, à la différence de l'insulte, peut refléter un état d'âme fait d'indignation, de colère ou d'irritation (Rouayrenc 1996), tandis que l'insulte est une attaque contre la personne (ibid.), constituant une menace pour la face ${ }^{10}$. La grossièreté qui constitue une menace est un élément correspondant à une certaine inversion de la hiérarchie, celle que l'on trouve dans le carnaval, où celui qui est haut placé dans la hiérarchie se retrouve en bas de l'échelle, et vice versa. Selon Bakhtine (1929), la vie du carnaval dévie le cours normal des choses et permet une inversion. Les lois, le respect, la politesse, et tout autre manifestation de l'ordre établi et de la hiérarchie, sont mis en cause. Ce genre d'attaques semblent être des contre-attaques visant un discours traditionnel hiérarchique. Mais font-elles partie d'un vrai débat, et apportent-elles de nouvelles idées ou solutions pour le bien commun?

A l'insulte se joint l'humour scatologique, dirigé contre le Président. Les exemples abondent, surtout sur le mode de mème et de gif. Par exemple un hippopotame faisant ses besoins.

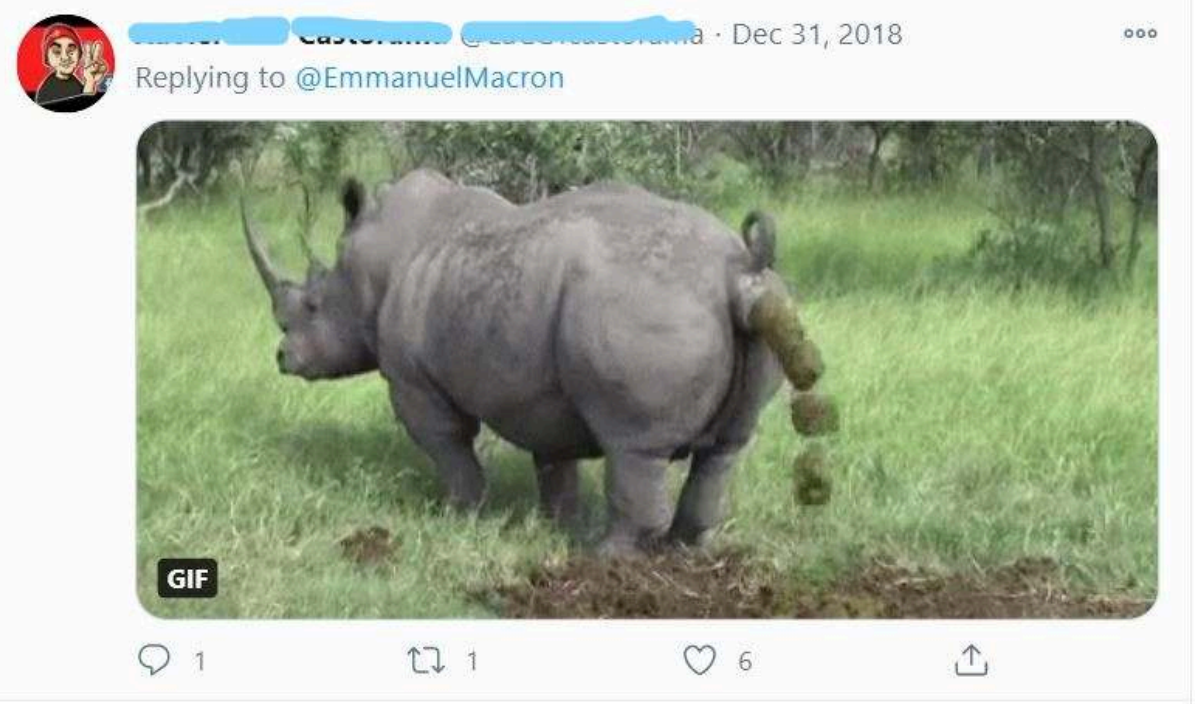

Rappelons que ce genre d'humour bas et vulgaire a été utilisé jadis dans la littérature à des fins satiriques (Bakhtine 1968). Ici, il s'agit même de diminuer personnellement l'autorité du Président afin de l'affaiblir. Ces attaques contre la personne peuvent coïncider avec l'atteinte à l'autorité institutionnelle : on porte atteinte à la personne en contestant sa légitimité, l'une des conditions de l'autorité institutionnelle, selon Bourdieu (1991 : 109). 

personnelle et institutionnelle. L'usage de la deuxième personne du singulier (« toi ») à la place de la forme de politesse («vous ») vise à montrer le manque de respect envers l'homme et envers la fonction : on réduit ainsi la distance qui le sépare des citoyens. En parallèle, il s'agit d'éliminer la hiérarchie qui s'exprime dans l'adresse "Monsieur le Président ». On peut y voir la mise en question de la légitimité et de la justesse de la hiérarchie (Arendt 1972 [1954]) qui sépare le Président de certains internautes. Le ton familier des messages, caractéristiques de ces réseaux (Herring 2007) donne cette impression d'ébranlement de la hiérarchie. Mais il s'agit d'un leurre car, en fin de compte, le Président n'entre pas en dialogue avec les usagers.

Certains exposent les manœuvres discursives qu'utilise Macron afin de persuader, en rajoutant des qualifications péjoratives se référant aussi à la façon dont le discours est prononcé (prononciatio) : locution trop soignée, usage de jolis mots, et carrément « manipulateur ». Les réactions reflètent pourtant des jugements contradictoires : pour certains Macron est un "mauvais acteur », pour d'autres il est un "menteur». Or, le menteur professionnel est un très bon acteur. Cette différence fondamentale est intéressante car il s'agit dans les deux cas de voix qui cherchent à le discréditer, mais les justifications de ces jugements péjoratifs sont contradictoires et loin d'être partagées par tous. réfèrent à une thèse de Macron. Ils la contredisent, ou montrent son manque de validité, en même temps qu'ils en exposent les non-dits. C'est là que réside la force de cette plateforme permettant une sorte de brainstorming. C'est là également que nous pouvons distinguer entre les réactions envers ce qui est dit dans le discours de Macron, et celles qui sont en rapport avec les non-dits de son allocution.

29 "foule haineuse ». Cet élément a été perçu par certains, et repris abondamment par d'autres, comme une délégitimation du mouvement des Gilets jaunes dans son ensemble. La reprise de cet élément (polémique en soi) a été radicalisée pour diaboliser celui qui l'a émis. Il est fort probable qu'il s'agit d'activistes anti-Macron ${ }^{11}$, qui profitent de la polémique qu'ils tentent d'exacerber. Rappelons cependant que Macron a parlé d'une colère "qui venait de loin ${ }^{12}$ pour montrer une certaine empathie avec le mouvement tout en reversant le blâme sur les anciens chefs de l'État présentés comme responsables de la situation. Parallèlement, nulle part dans le discours il n'a mentionné explicitement les Gilets jaunes. Ainsi le discours de Macron laisse-t-il un flou qui permet une interprétation de l'élément "foule haineuse " comme incluant tous les Gilets jaunes, et pas seulement les manifestants les plus violents. Des utilisateurs, éventuellement des activistes politiques, ont immédiatement repéré ce qui est devenu une formule (Krieg-Planque 2015), à savoir «la foule haineuse», en retournant le propos de Macron contre lui. Cela aboutit à des critiques dénonçant un Président incapable d'écouter le peuple. Ces réactions usent d'éléments polémiques, de l'ironie, des attaques ad personam et ad hominem.

Le manque d'écoute est une critique récurrente qui affaiblit la légitimité accordée à Emmanuel Macron en ce qu'elle le montre en quelque sorte détaché du peuple. A partir du moment où il n'écoute pas les Français alors qu'il est censé les représenter, le Président perd sa légitimité aux yeux d'un certain nombre d'internautes. S'ensuit un affaiblissement de sa capacité à agir sur ces auditoires; son autorité s'en trouve 
affaiblie. Cette perte d'autorité se manifeste clairement dans les attaques ad hominem dirigée contre Macron.

31 L'ad hominem consiste dans la contradiction relevée entre le discours et la réalité. Cet ad hominem revient sous différentes formes : contradiction entre les paroles et la réalité, entre les paroles et les actes (argument Tartuffe, selon Gauthier 1995). Pointer du doigt ce décalage constitue une stratégie de menace pour la face (Jucker 1986) ${ }^{13}$. Par exemple, @passionsdelisah affirme avoir cessé d'écouter le discours au bout de quelques minutes en raison du manque d'écoute dont a fait preuve Macron: «Vous ne voyez rien et entendez rien de ce que vous disent les citoyens ?...»

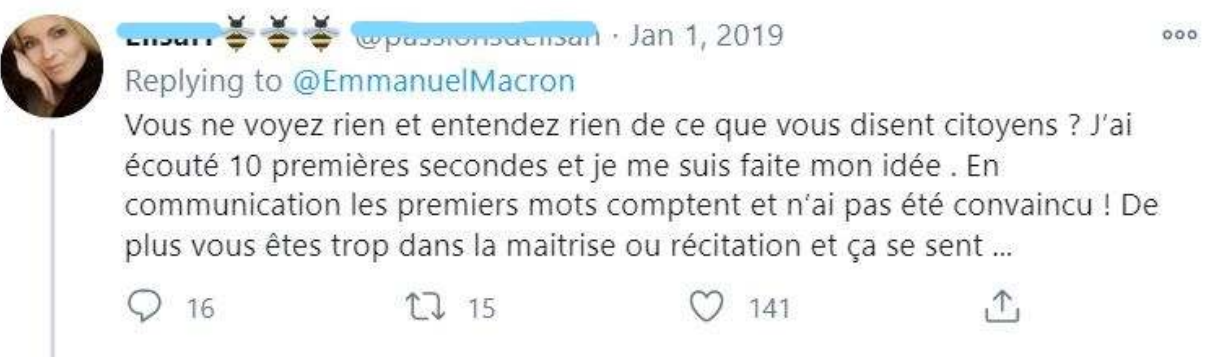

Ou encore @BlamartEmilie qui dit «Vous êtes méprisable et méprisé. Vos vœux vs pouvez les garder pour votre petit nombril. Vous n'écoutez pas le peuple, vous le méprisez comme jamais.» Le décalage entre le discours qui fait abstraction des exigences des protestataires, selon ces réactions, et la réalité, rendrait Macron illégitime et saperait son autorité.

D'autres réactions mettent l'accent sur le fait que le Président est détaché de la réalité des Français. En témoignent les propos suivants venant de @annaboquet5 : «Quand on l'écoute, tout va bien. Pourquoi le peuple est dans la rue alors!» Une autre variation est celle qui (faisant allusion aux résultats des élections - on prétend qu'il a été élu par défaut), utilise le topos de la quantité pour marquer que le Président ne comprend pas les revendications du mouvement social qui est plébiscité par la majorité : «les gilets jaunes ont plus de $80 \%$ d'opinions favorables de la part de toute la population électorale Fr vous êtes sous les $20 \%$ d'opinions favorables de toutes la population électorale ».

La figure de l'ironie est utilisée abondamment, également en relation avec l'affirmation de «foule haineuse ». @Asuba 2013 affiche un mème montrant un Gilet jaune qui prend dans ses bras un policier. 
moun............ @isurvacuTs . Jan 1, 2019

Replying to@EmmanuelMacron

L'image effroyable de cette FOULE HAINEUSE de Gilets Jaunes! twitter.com/leGneral2/stat...

Ca fait froid dans le dos.

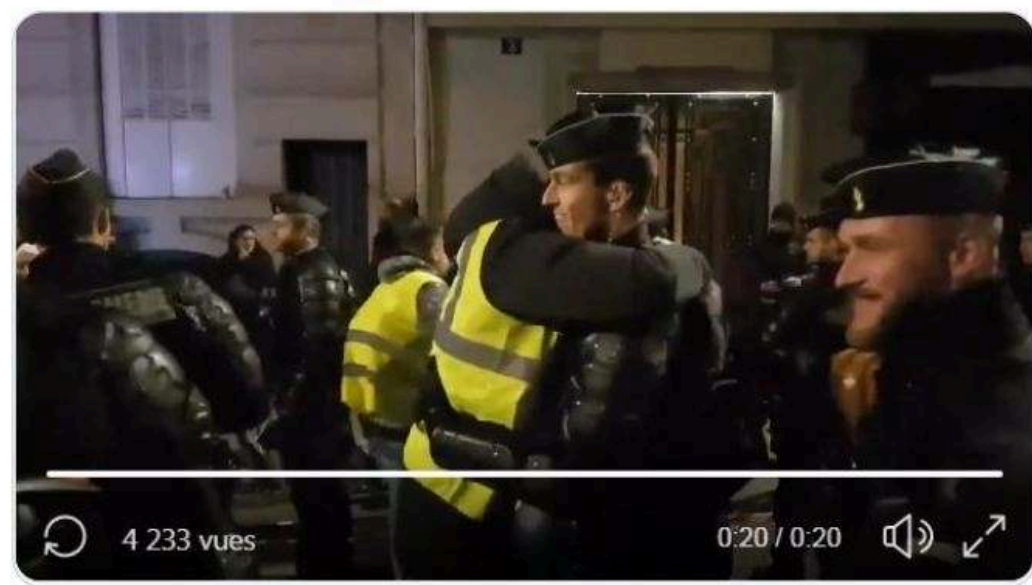

This Tweet is unavailable.

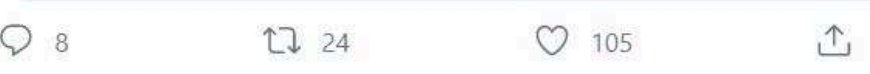

Image sympathique d'un Gilet jaune qui peut susciter une identification avec le mouvement, contrairement à la façon dont Macron les caractérise dans son discours. Ce même utilisateur ajoute une inscription procédant par ironie ${ }^{14}:$ " L'image effroyable de cette FOULE HAINEUSE de Gilets Jaunes ! [...] Ça fait froid dans le dos. ». La qualification d'« effroyable » décrivant le sens contraire de ce que l'on voit dans l'image, produit de l'ironie (par antiphrase), cherchant à ridiculiser les propos de Macron.

Rappelons néanmoins qu'il n'a pas visé dans cet énoncé tous les Gilets jaunes : il a caractérisé les plus violents, en parlant des porte-voix. Or, en dépit de ces spécifications, cet énoncé a été mal reçu, comme le montrent aussi d'autres réactions. Celle de @philippe_barthe : « C'est quand même inquiétant un Président qui pense que la révolution de 1789 était motivée par la haine et non par un besoin de justice, de démocratie et l'abandon des privilèges, je me demande si les cours à l'ENA il n'y a pas un problème! » Il s'agit d'un exemple historique qui fait référence à un événement connu du passé (Amossy 2000 : 24) : on met en relation le mouvement des Gilets jaunes avec la Révolution française, afin de créer une association indéniable, une analogie entre le phore, l'élément connu (la Révolution) et le thème (le mouvement GJ). Remarquons que ce procédé analogique utilisé par les internautes vise, tout comme chez les politiques, à la construction d'un discours d'autorité, qui ne laisse pas place à l'opposition. Si les Gilets jaunes sont du côté des révolutionnaires, Macron est donc du côté des royalistes - ce qui jette sur lui un discrédit total dans une République.

La contradiction entre les propos du Président et la réalité se manifeste dans la présentation de la «foule haineuse" comme incarnant la solidarité et la chaleur humaine, et comme manifestant paisiblement. @Opuscopus21 affiche une vidéo où l'on voit des manifestants paisibles en affirmant que BFMTV ne la diffuse pas, mettant en cause, en passant, la fiabilité de la chaîne d'informations en continu. La possibilité de rajouter un fichier vidéo, caractéristique de ce réseau, a fortiori à la lumière de la contrainte de brièveté, peut contribuer à renforcer le message. @Calam108 écrit : 
Très intelligent de parler de personnes comme d'une foule haineuse alors qu'on côtoie des gilets jaunes, voisins, commerçants, généralistes, famille parfois qui n'ont aucunement ce profil. Un mépris de plus, un discrédit de plus, un pas de plus dans la non confiance en vous. de sa formulation sur un mode affirmatif, façon «donneur de leçons» (« ne pas oublier " $)^{16}$, à cause du thème de la " vérité ", et de la paraphrase du slogan " Travailler plus pour gagner plus ». L'affirmation : « On ne peut pas travailler moins, gagner plus » vise à répondre aux revendications des manifestants Gilets jaunes. Elle est une variation du slogan, utilisé par Nicolas Sarkozy dans sa campagne en 2007, qui vise à clore tout débat et faire autorité (Krieg-Planque 2012 : 105) en ce qui concerne le besoin de travailler plus. Indirectement la responsabilité d'améliorer leur propre situation revient aux citoyens. Le slogan peut exercer une force d'influence qui tient à son caractère de " cela va de soi », car il s'agit d'un lieu commun de la pensée libérale. Or, les internautes détectent ces éléments de langage et y réagissent violemment, comme @Marcel_Renou : «Je vous souhaite une destitution en 2019, car votre cynisme n'a que trop duré: oser sous entendre que le chômage est la faute du chômeur un 31 déc. résume parfaitement tout. Si fort que vous vous accrochez, le peuple vous délogera! » parole est ambiguë et dont le mensonge a bien été révélé au sujet de Benalla. @letouslois écrit : « Vœu de vérité. Il fallait le trouver celui-là !» 
lalevée georges@letoulois.Dec 31, 2018

Replying to@EmmanuelMacron and@Elysee Vœu de Vérité il fallait le trouver celui-là

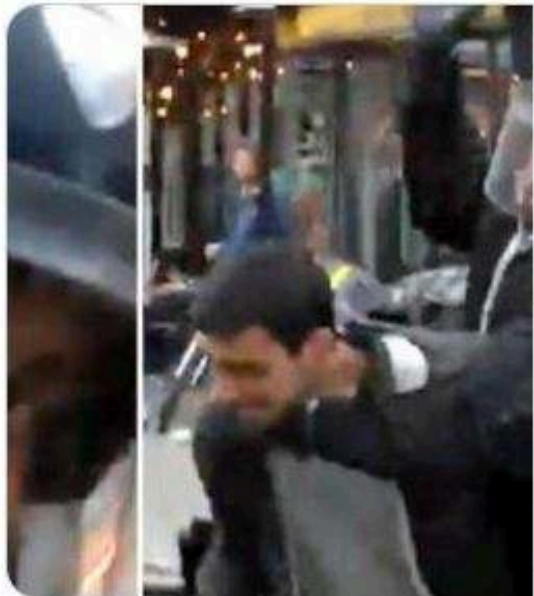

4

〔】 11

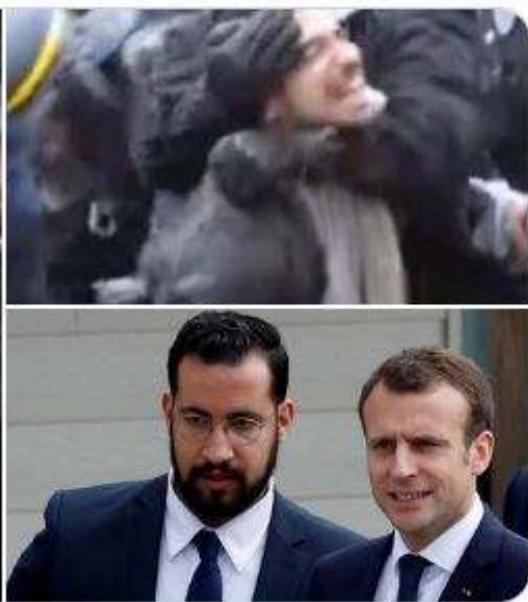

O 114

个 autre photo de Benalla frappant un manifestant. Une fois de plus, un mème sert à conférer une crédibilité aux propos contestataires d'un utilisateur, et en même temps à porter atteinte à la parole du Président. Un vœu de vérité qui est émis par celui qui fait la démonstration du contraire, n'est pas crédible. Son ethos montré (Maingueneau 1996) est ici la source de l'affaiblissement de son autorité.

L'attaque ad hominem peut recourir aussi à la qualification péjorative de «menteur » en montrant la contradiction entre les paroles de Macron et ses actes. @hamani_claude écrit : «On ne peut rien construire dans le mensonge' si ça ce n'est pas nous prendre pour des cons... rien que l'affaire BENALLA MENTEUR ».

Dans ce cas, il ne s'agit pas seulement d'insulter Macron, ou de le disqualifier, ce qui serait une attaque ad personam (comme dans le cas mentionné plus haut dans la section correspondante). L'internaute expose la contradiction entre le vœu de vérité et un élément non-dit de son discours. Notons que les lettres en majuscules donnent une tonalité de colère à la fin du message, équivalente à un cri - une variation de l'hybridité oral/écrit, caractéristique des écrits numériques (Marcoccia 2016 : 11). policière. L'argument sous-jacent est qu'en démocratie, la force légitime doit être justifiée et mesurée. Les internautes joignent des documents qui, s'ils sont authentiques, peuvent fragiliser la légitimité du Président. 
vEdT\% je(u)di @ET_Id883_1.Dec 31,2018

Replying to@EmmanuelMacron

La \#fraternité selon \#Macron:

twitter.com/J0sephch/statu..

\#ministèredelaverité \#Orwell \#GiletsJaunes

C Joseph IIS A @ J Osephch.Dec 31,2018

4 sos Notre grand leader \#Macron vous souhaite une violente année 2019 et une belle répression policière:

4 Tabassages, matraquages, flashball, gazage, yeux crevés, machoirs cassées, mains coupées..

Happy New year

\#France \#ViolencesPolicières \#VoeuxMacron

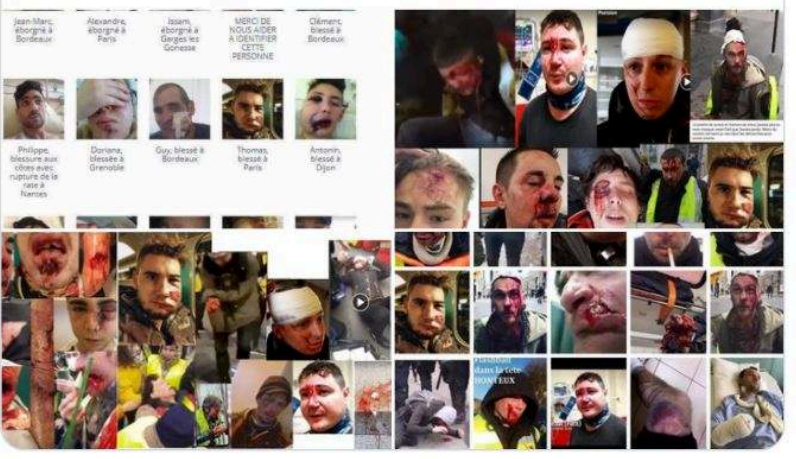

Q 2 †】

O 11

个

Il est peu probable que les usagers aillent vérifier leur authenticité, en raison du caractère éphémère des messages sur les réseaux (Herring 2007), et de la vitesse à laquelle les messages sont dépassés. Des transgressions policières ne sont pas mentionnées dans son allocution, alors qu'il félicite les forces de l'ordre, les gendarmes et les policiers. Ce manque d'équilibre dans son allocution entre les forces de l'ordre (félicités) et les manifestants (délégitimés), ouvre la voie à un nombre de réactions qui affaiblissent son autorité en tant que chef de l'État juste dans un état de droit, comme celle de @AjAdeline qui écrit :

Adeline Jaouadi@AjAdeline.Dec 31, 2018

Replying to@EmmanuelMacron

Des voux attiseurs de haine, innover avec les mêmes qui refusent et nous mentent sur les taxes, les lois, défend uniquement des intérêts privés et minoritaires non en FRANCE l'état de droit n'existe plus et vous le confirmer en autorisant la violence des forces de l'ordre

Qui plus est, il s'agit d'exposer le manque de cohérence entre les propos du Président et les caractéristiques de son allocution de Nouvel An. Au lieu de répondre à l'exigence d'un discours rassembleur, il présente un discours de division. @SarahlLoire écrit aussi dans le même esprit que la réaction ci-dessus: «Des vœux? Juste de la politique politicienne sans émotion ni empathie [...] Vous aimez peut être la France, mais le peuple [...] J'espérais un discours de réconciliation pour apaiser le pays. Ni gilet jaune ni en marche, Chirac me manque aujourd'hui. »

Le besoin d'un chef, d'une autorité capable de réconcilier, se retrouve dans un certain nombre de réactions de ce genre. @LaetitiaMaeren propose d'apprendre chez Chirac comment présenter ses vœux : «Tenez, écoutez un vrai chef d'Etat présenter ses vœux aux Français, prenez des notes et tachez d'être à la hauteur du job en 2019. http:// 
m.youtube.com/watch?v=1QfD2pJt5U » [45] Comme réaction à ce tweet, un deuxième utilisateur écrit : « le dernier Président ».

On voit bien que l'autorité personnelle de Macron est mise en cause, mais non pas l'autorité présidentielle en tant que telle. Ces internautes rappellent les modèles d'autorité auxquels ils aspirent : dans ce cas il s'agit de Jacques Chirac ${ }^{17}$. Dans un autre, c'est un mème sur lequel figurent De Gaulle, très grand, et à ses côtés Macron comme un nain.

\section{Dynamique de la conversation : autorité personnelle vs. institutionnelle}

50 Il est possible de distinguer deux aspects différents de la dynamique sur la toile : (1) rapport entre des internautes qui coopèrent et se légitiment l'un l'autre, (2) rapport entre les internautes qui s'opposent. Il est rare de trouver une réaction qui n'est ni de soutien ni d'opposition. Chaque type de rapport peut comporter une panoplie de réactions. Nous voudrions ici nous attarder sur deux exemples, qui représentent chacun un type de rapport.

Le premier cas montre un soutien entre internautes. Rappelons l'exemple mentionné au sujet de l'ironie («L'image effroyable de cette FOULE HAINEUSE... »). Celle-ci, pour être efficace, postule un certain accord parmi les membres d'un groupe, ce qui constitue son caractère social (Perelman et Olbrechts-Tyteca [1958] 2008 : 280). Les éléments qui sont acceptés par tout un groupe (celui des manifestants et de leur soutiens) sont exposés par le mème (la photo du Gilet jaune sympathique). L'ironie devient donc accessible (le décalage est saillant) à tous grâce au recours à des éléments visuels caractéristiques de cette plateforme. Et en effet, @anmary5966 renforce la critique: "Oui il faut montrer aussi les bons côtés». Cette internaute se montre modérée et en quête de justice, renvoyant par opposition l'image d'un Président manquant de neutralité. Dans cette dynamique, les réactions se renforcent mutuellement. La complémentarité des réactions affaiblit encore plus la légitimité de Macron.

Le deuxième cas est celui d'un rapport d'opposition entre utilisateurs, voire de polémique. 
Iiliade@rtipaphy·Dec 31, 2018

Replying to@EmmanuelMacron and@Elysee

Je me demande si ce con s'est pas fait briefer par Stéphane Bern, il a les mêmes intonations.

Quant au baratin, il a dû lui être fourni par Benalla. \#MacronDemission \#Macron2OH

Q 1

〔】 2

O 17

Steph@noSteph.Jan 1,2019

Le Respect en PLS

Sérieux "ce con" c est le président de la République Française.

En accord ou pas avec sa politique c est votre droit. Mais un minimum de respect ne serais ce que pour la fo ction si ce n'est pas pour I homme.

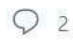

$\uparrow\urcorner$

○ 1

个

Rêvons Lution @RevonsLution·Jan 1,2019

Le respect c'est dans les deux sens. II n'y a pas de respect à avoir pour un élu qui crache verbalement à la tête du peuple, et c'est valable d'un conseiller municipal au president de la république. On reverra la notion de respect quand il arrêtera de nous prendre pour des cons !

Q 1

$\uparrow \downarrow$

O 1

Steph@noSteph.Jan 1,2019

c est sa politique qui est irrespectueuse ? Savez vous toutefois que "ce con" à été élu démocratiquement en annonçant tout ce Qu il allait faire. détesté ou non il a la fonction. Débattre de sa politique OK, mais hurler sans admettre que I autre peut avoir raison c du dictat.

Q 3

$\uparrow \downarrow$

O

@rtipaphy écrit : « Je me demande si ce con s'est pas fait briefer par Stéphane Bern, il a les mêmes intonations. Quant au baratin, il a dû lui être fourni par Benalla \#MacronDemission \#Macron20H ». Ce à quoi répond @noSteph : "Le Respect en PLS Sérieux 'ce con' c est le Président de la République Française. En accord ou pas avec sa politique c est votre droit. Mais un minimum de respect ne serais ce que pour la fonction si ce n'est pas pour 1 homme ». Pour cet internaute, l'atteinte à la personne d'Emmanuel Macron est en fait une atteinte à l'autorité institutionnelle : on ne peut pas insulter sa personne sans dégrader la fonction présidentielle. Cette réaction est dans l'esprit de la notion des « deux corps du roi » (Kantorowicz 1997). Ainsi, les ad personam sont aussi des attaques sur l'autorité institutionnelle. @rtipaphy écrit : 
Iiliade@rtipaphy·Jan 1,2019

Replying to@noSteph@EmmanuelMacron and @Elysee

Ceconnard@EmmanuelMacron est irrespectable tant il bafoue lui même le peuple, les institutions et sa fonction ds sa manière de l'incarner.

C'est bien parce que j'ai du respect pour la fonction que je n'accepte pas qu'un opportuniste aux dents longues en fasse ce qu'il en fait.

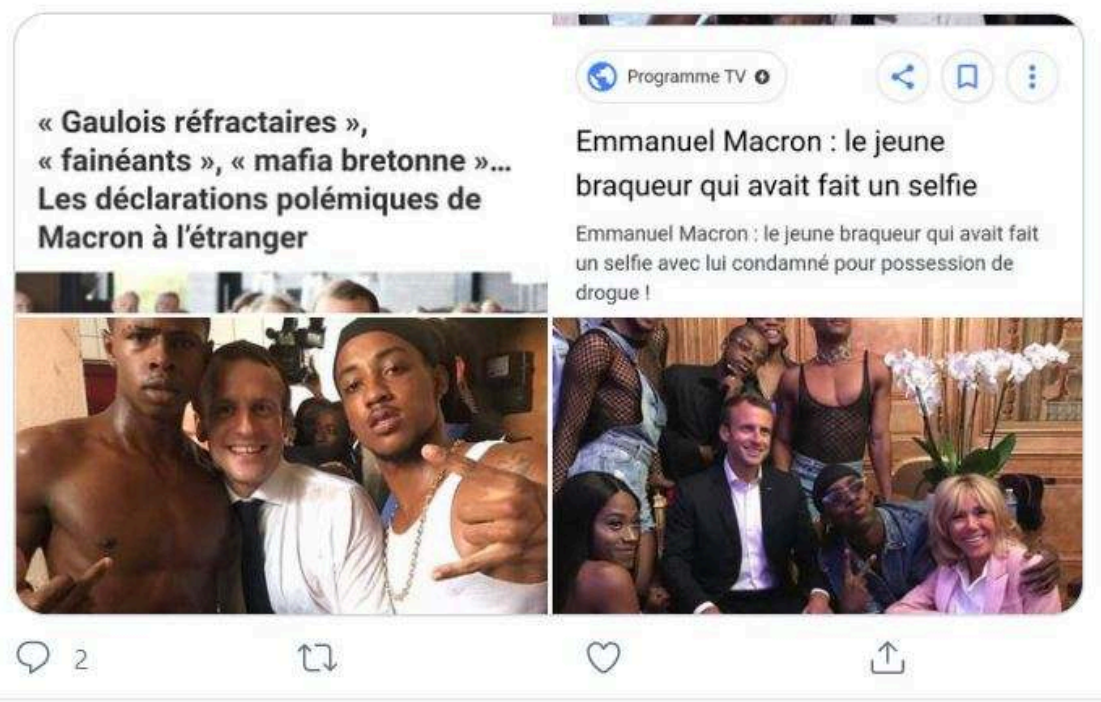

Cette réaction est accompagnée de photos de Macron à la fête de la musique avec les jeunes torse nu, et le selfie avec le jeune braqueur de Saint Martin. Cette réaction semble vouloir procéder par un facework: il s'agit d'abord d'aller davantage vers l'extrême, par l'utilisation de la grossièreté (le con devient « connard»). Ensuite, elle établit une distinction entre la position (le statut) et la personne, contrairement à l'interlocuteur. Elle explicite son respect envers l'autorité institutionnelle en soi impersonnelle (qui relève des lois), et dénonce l'abus que la fonction subit avec une personne considérée comme illégitime en raison d'un comportement défini comme défectueux. Enfin, elle appuie son jugement sur des documents visuels. $@$ @oSteph continue en lui disant :

$\mathrm{j}$ ai aucun problème $l$ vous dire $\mathrm{Qu}$ il fait mieux le job que vous par exemple. parce que gérer un état $\mathrm{c}$ est plus compliqué que gueler sur Twitter sous le couvert de 1 anonymat. Si vous êtes pas contente engager vous en politique et changer les choses.

Il met ainsi en évidence les caractéristiques de Twitter qui permettent à tout un chacun de s'exprimer publiquement sans avoir contribué significativement à la gestion du pays. En plus, il utilise un procédé qui vise à la faire taire: soit elle s'engage en politique, soit elle s'abstient de prendre la parole. C'est là que la réaction devient plus polémique en attaquant personnellement @rtipaphy. Cependant, elle lui répond en mettant l'accent sur le manque de pertinence de son argumentation: «Vous avez pas répondu à ma question et vs comparez 2 personnes [voulant dire : un homme politique et un usager de Twitter. K.S.] ds des situations différentes ce qui est aberrant. ». Dans les trois 'tours d'écriture' (Marcoccia 2016) qui s'ensuivent, chacun dirige vers l'autre un mélange d'attaques ad personam et ad hominem bien argumenté.

Cette interaction reflète un mécanisme d'autorégulation des normes de conduite par les internautes. Ceux-ci réagissent et critiquent les invectives et les grossièretés. Cela peut être la raison pour laquelle certains d'entre eux cherchent à se légitimer et à 
éviter une menace pour leur propre face (surtout ceux qui affichent publiquement leur page, leur motto, et d'autres informations qui les identifient). L'ad hominem sert à montrer un fonctionnement incohérent, et donc justifie l'attaque ad personam.

\section{Les soutiens d'Emmanuel Macron usent-ils de procédures différentes?}

Les réactions contenant des évaluations positives à l'allocution de Macron signalent la justesse, la beauté, la positivité du message, légitimant ainsi l'autorité du Président. Comme dans la réaction de @GEchevarria : «Bravo pour ce message juste et positif ! Il s'agit là de la catégorie de l'évaluation morale mentionnée chez Van Leeuwen comme l'un des fondements de la légitimation. Ceux qui apprécient l'allocution mentionnent les qualités positives du discours épidictique, presque sans référence au contexte politique du moment. D'autres congratulent le Président à leur tour, en un acte de coopération. Ces vœux de bonne année peuvent figurer sous la forme d'un mème. Si c'était une interaction en face-à-face, on aurait pu parler de face flaterring act (KerbratOrecchioni 2005), qui permet de sauvegarder la face de Macron, ainsi que de renforcer son autorité. D'autres réagissent aux émotions que ce discours suscite en eux. Et il y a ceux qui rassemblent les trois types de réactions légitimant le discours, comme @katiasoutienEM (qui déclare ouvertement son soutien à Macron): «Merci pour ce magnifique discours. Quelle hauteur!! Je suis tellement fière d'avoir voté pour vous. Tous mes vœux pour cette nouvelle année ». Ou celui de @Anne75005 :

Anne Vicher@Anne75005.Dec 31, 2018

Replying to@EmmanuelMacron

Bravo Président Votre discours de conviction de fermeté et d espoir rappelant à chacun ses droits et ses devoirs nous redonne confiance pour défendre nos valeurs en 2019 @LaREMParis

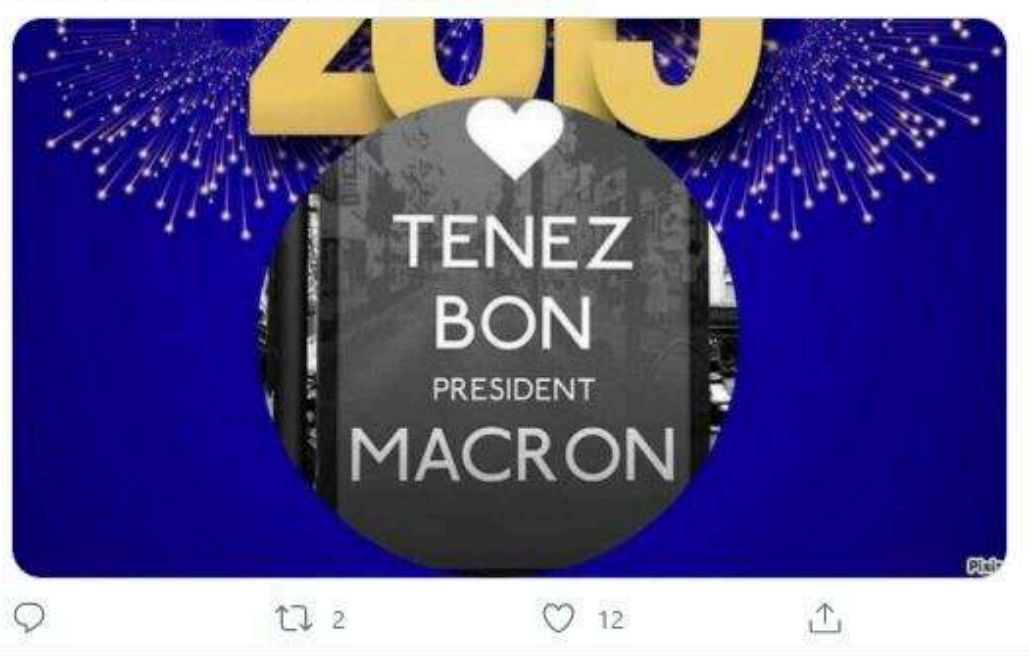

\section{Conclusion}

Les réactions se divisent de façon tranchée entre ceux qui renforcent l'autorité du Président Macron et ceux qui la contestent. Les premiers obéissent au principe de coopération, présentant des vœux en retour ; ils louent la beauté de cette allocution et mettent en valeur son caractère positif. Selon les réactions contestataires, l'ethos 
discursif de l'orateur ne parvient pas à susciter la confiance en raison du manque d'adéquation entre paroles et réalité, et entre paroles et actes. C'est l'autorité personnelle qui est ébranlée par leurs attaques. Leurs réactions sont fondées sur deux types d'argument: ad personam et ad hominem, qui se présentent de façon variée et créative sous divers modes : verbal, visuel (les mèmes, les photos) et sonore (chanson parodique). Les deux types d'argument sont parfois entremêlés: l'ad hominem pointant du doigt les incohérences et les contradictions dans le fonctionnement $d u$ Président - sert à légitimer les attaques ad personam (y compris les invectives). Les internautes exposent les manipulations verbales et les non-dits du discours. De ce point de vue, les usagers nourrissent une conversation qui aiguise la conscience civique.

Cependant, en parallèle, l'instrumentalisation de la conversation par les activistes politiques est saillante. Certains le déclarent explicitement, mais le problème est que nous ne connaissons pas le nombre de ceux qui opèrent sous un pseudonyme. Le contexte politique précis de cette allocution joue un rôle prépondérant: les protestataires - surtout les adversaires politiques - y trouvent avant tout un moyen de disqualifier Macron en prenant ce discours comme prétexte. Cela explique les "solutions " auxquelles ils ont recours : soit on se défoule, soit on attise la polémique, soit on désigne des adversaires politiques pour remplacer Macron - signes de la présence d'activistes sur le réseau. Il s'agit d'atteintes à l'autorité personnelle et institutionnelle du Président ainsi que d'une remise en question non pas des lois de la République (l'autorité impersonnelle), mais de l'idéologie libérale.

Quant à la dynamique de la conversation, nous avons vu que la plateforme permet d'affaiblir l'autorité du Président grâce au soutien mutuel que se prêtent les internautes. En parallèle, des conversations d'usagers qui s'opposent entre eux incitent chacun à justifier ses propres propos, et à attaquer ceux du camp opposé. Par ailleurs, il existe dans la dynamique de la conversation des éléments qui sont propres aux interactions en face-à-face: des menaces pour la face, du travail de «figuration ». Néanmoins, les menaces que les usagers utilisent entre eux sont beaucoup moins violentes que celles dont ils font usage pour faire perdre la face à Macron. La dynamique qui se manifeste entre les opposants nous montre qu'il existe des mécanismes d'autorégulation, qui sont avantageux pour la vie démocratique.

61 En tous cas, les réactions montrent un décalage entre l'acte perlocutoire des vœux et l'effet qu'il suscite: la plupart de ceux qui commentent l'allocution la contestent violemment (il est vrai que se pose la question des motivations de ces commentateurs). Ces enchaînements de réactions violentes sont comparables aux cheveux tentaculaires de la Méduse, créature mythologique à laquelle le héros Persée peut se confronter au moyen d'un miroir. Twitter en constituerait un équivalent moderne. Macron, comme Persée, n'est pas confronté directement aux regards pétrifiants de la tête aux milles serpents : c'est le medium qui le sauve (ou bien, le mettrait-il au contraire davantage en danger?) 


\section{BIBLIOGRAPHY}

Amossy, Ruth. 2014. Apologie de la polémique (Paris : PUF)

Amossy, Ruth. 2000. L'argumentation dans le discours. Discours politique, littérature d'idées, fiction (Paris : Nathan)

Arendt, Hannah. 1972 [1954]. « Qu'est-ce que l'autorité ? », La crise de la culture. (Paris : Gallimard) Austin John, Langshaw. 1970. Quand dire, c'est faire : How to do things with words (Paris : Seuil) Bakhtine, Mikhail. 1968. Rabelais and His World (Cambridge: MIT Press)

Bourdieu, Pierre. 1991. «Authorized Language ", Language and Symbolic Power. B. Thompson, trans. G. Raymond \& M. Adamson (ed.). (Cambridge: Polity Press)

Brown, Penelope \& Steven C. Levinson. 1987 [1978]. Politeness. Some Universals in Language Usage (Cambridge: Cambridge U. P.)

Candel, Etienne \& Pergia Gkouskou-Giannakou. 2017. « Autorité et pratiques de légitimation en ligne ", Quaderni 93.2 (Paris : FMSH)

Davison, Patrick. 2012. «The Language of Internet Mèmes » The Social Media Reader, (ed.) Michael Mandiberg, NYU Press, 120-134. http://www.veryinteractive.net/content/2-library/46the-language-of-internet-memes/davison-thelanguageofinternetmemes.pdf (consulté le $1 / 4 / 2019)$

Fetzer, Anita \& Peter Bull. 2012. « Doing leadership in political speech: Semantic processes and pragmatic inferences », Discourse \& Society, 23. 2, 127-144

Gauthier, Gilles. 1995. «L'argument périphérique dans la communication politique : le cas de l'argument “ad hominem", Hermes 16, Argumentation et rhétorique, 167-185

Groarke, Leo. 2017. « Editorial Cartoons and ART Arguing with Pinocchio », Argumentation in context 14, « Multimodal Argumentation in Media Genres », (Amsterdam: Benjamins), 81-109 https://doi.org/10.1075/aic.14.04gro (consulté le 1/4/2019)

Grundlingh, Lezandra. 2018. " Memes as speech acts", Social Semiotics 28.2, 147-168 https:// doi.org/10.1080/10350330.2017.1303020 (consulté le 1/4/2019)

Herring, Susan C. 2007. «A Faceted Classification Scheme for Computer-Mediated Discourse », Language\&Internet 4 http://www.languageatinternet.org/articles/2007/761

Jucker, Andreas H. 1986. News Interviews: A pragmalinguistic Analysis (Amsterdam : Benjamins)

Kantorowicz, Ernst Hartwig. 1997. The King's two bodies: A study in Medieval Political Theology (New Jersey : Princeton U. P.)

Kerbrat-Orecchioni, Catherine. 2005. Le discours en interaction (Paris : Colin)

Krieg-Planque, Alice. 2015. « Construire et déconstruire l'autorité en discours. Le figement discursif et sa subversion », Mots. Les langages du politique 107, 115-132 https://doi.org/10.4000/ mots.21926 (consulté le 18/3/2020)

Leblanc, Jean-Marc. 2016. Analyses lexicométriques des voeux Présidentiels (London: ISTE Editions) Longhi, Julien. 2013. "Essai de caractérisation du tweet politique ", L'information grammaticale 136, 25-32 https://halshs.archives-ouvertes.fr/halshs-00940202 (consulté le 18/3/2020) 
Longhi, Julien. 2019. « Le projet \#Ideo2017 : quelle implication du / de la chercheur-e en tant qu'acteur-trice potentiel du changement social ? Exemplification à partir du discours politique », Cahiers de Linguistique, 442 https://halshs.archives-ouvertes.fr/halshs-02153102 (consulté le $18 / 3 / 2020)$

Maingueneau, Dominique. 1996. Les termes clés de l'analyse du discours. (Paris : Seuil)

Marcoccia, Michel. 2016. Analyser la communication numérique écrite (Paris : Colin)

Mayaffre, Damon. 2012 [2004]. Le discours Présidentiel sous la Ve République. (Paris : Presses de SciencesPo)

Oger, Claire \& Michèle Monte. 2015. « La construction de l'autorité en contexte. L'effacement du dissensus dans les discours institutionnels », Mots. Les langages du politique 107, 5-18 https:// doi.org/10.4000/mots.21847 (consulté le 18/3/2020)

Paveau, Marie-Anne. 2006. Les prédiscours : sens, mémoire, cognition (Paris : PUF)

Paveau, Marie-Anne. 2013. « Genre de discours et technologie discursive. Tweet, twittécriture et twittérature », Pratiques 157-158, 7-30. https://doi.org/10.4000/pratiques.3533 (consulté le $18 / 3 / 2020)$

Perelman, Chaim \& Lucie Olbrechts-Tyteca. 2008 [1958]. Traité de l'argumentation (Bruxelles : Édition de l'Université de Bruxelles)

Revault d'Allonnes, Myriam. 2012. La crise sans fin. Essai sur l'expérience modern du temps (Paris : Seuil)

Rouayrenc, Catherine. 1996. Les gros mots (Paris : PUF, « Que sais-je ?»)

Sadoun-Kerber, Keren. 2018. « Gestion et réparation d'image : Emmanuel Macron dans

"L'Emission politique" ", Langage \& Société 164, 75-95

Sebbah, Brigitte Lucie Loubere et al. 2018. « Les Gilets jaunes se font une place dans les médias et l'agenda politique », Rapport de recherche 7.12.2018 (Toulouse LERASS) https://halamu.archives-ouvertes.fr/hal-02120478 (consulté le 18/3/2020)

Shifman, Limor. 2013. « Memes in a Digital World: Reconciling with a Conceptual Troublemaker ", Journal of Computer-Mediated Communication 18, 362-377 https://doi.org/10.1111/ jcc4.12013 (consulté le 18/3/2020)

Van Leeuwen, Theo. 2007. « Legitimation in discourse and communication », Discourse \& Communication, 1.1, 91-112 https://journals.sagepub.com/doi/10.1177/1750481307071986

Wagener, Albin. 2020. « Mèmes, gifs et communication cognitive-affective sur Internet. L'émergence d'un nouveau langage humain », Communication 37.1, https://doi.org/10.4000/ communication.11061 (consulté le 18/3/2020)

\section{NOTES}

1. https://twitter.com/emmanuelmacron/status/1079814545873481729?lang=en

2. À la fois au sens de conversation et de débat.

3. Susan Herring (2007) formule un modèle d'analyse de discours médié par ordinateur (CMDA) qui comporte deux séries de catégories: la première contient dix facteurs technologiques: synchronicité, mode de transmission, durée du message archivé, taille, anonymat vs. pseudonyme, et autres. La deuxième série contient des facteurs liés à la situation de la 
communication: cadre participatif, participants, objectifs, cadre thématique, ton, activités, normes, code langagier.

4. Les utilisateurs de Twitter peuvent être considérés comme des participants au deuxième degré ("second frame participants" (Fetzer 2013 : 3)) tandis que le Président Macron est lui "first frame participant » (ibid.). Il s'agit de l'interaction qui s'établit entre l'homme politique (Emmanuel Macron, en l'occurrence) avec un auditoire qui visionne la vidéo, mais qui n'est pas son auditoire immédiat.

5. Ce, à la différence d'un Donald Trump, par exemple, chez qui le caractère spontané est bien présent.

6. La philosophe Gloria Origgi (2015) distingue entre l'autorité épistémique et l'autorité politique des hommes politiques, en montrant, entre autres, l'utilisation des scientifiques par les hommes politiques à leurs propres fins. Contrairement à la conception émise par Van Leeuwen, l'expertise, selon Origgi, est par définition autoritaire (160). Elle n'est pas en perte d'autorité et pour cette raison elle entretient des rapports problématiques avec la démocratie.

7. Exemples du discours de Macron: "Il serait dangereux que notre situation nous conduise à ignorer le monde qui nous entoure [...]» (mes italiques).

8. Selon Fetzer et Bull (2012), il y a quatre catégories de verbes qui se traduisent en leadership (verbes d'évènement/action, verbes d'intention, verbes de subjectivation et verbes de communication), parmi elles les verbes d'évènements, et les verbes d'intention, abondamment utilisés par Macron « Nous voulons », etc.

9. Ou argument ad hominem de type personnel, selon Gauthier (1995: 178) qui distingue trois types d'ad hominem.

10. Le terme de «menace pour la face » (Goffman 1967, Brown et Levinson 1978) tiré de l'analyse des interactions est pertinent, même s'il s'agit d'interaction virtuelle, et non en face-à-face. Car les attaques présentent une menace pour la face " positive » de Macron, dans la façon dont celuici cherche à se montrer. En revanche, il n'y a pas de menace réelle sur la face «négative ", qui consiste dans la liberté d'expression, d'occupation d'espace, etc. car les internautes ne peuvent pas maîtriser l'activation principale de la vidéo (ils peuvent la manipuler individuellement). En revanche, les réactions montrent un besoin de menacer aussi la face négative (comme des exemples de « chut! » ou « tg »)

11. Certains mentionnent explicitement leur appartenance à un parti politique.

12. «Mais nous avons aussi vécu de grands déchirements et une colère a éclaté, qui venait de loin ; colère contre les injustices, contre le cours d'une mondialisation parfois incompréhensible ; colère contre un système administratif devenu trop complexe et manquant de bienveillance ; colère aussi contre des changements profonds qui interrogent notre société sur son identité et son sens. "

13. Cette stratégie est pertinente ici aussi bien qu'il s'agisse d'interaction qui n'est pas en face-àface.

14. Rappelons que par l'ironie « on veut faire entendre le contraire de ce qu'on dit » (Dumarsais, cité dans Perelman et Olbrechts-Tyteca 2008 : 279).

15. «D'abord un vœu de vérité. Oui, nous souhaiter en 2019 de ne pas oublier qu'on ne bâtit rien sur des mensonges ou des ambiguïtés. Or, je dois bien dire que depuis des années, nous nous sommes installés dans un déni parfois fragrant de réalité. On ne peut pas travailler moins, gagner plus, baisser nos impôts et accroître nos dépenses, ne rien changer à nos habitudes et respirer un air plus pur!»

16. Cette impression est évoquée à l'aide d'autre moyens comme les phrases impersonnelles, l'effacement énonciatif (Rabatel 2004), ainsi qu'une multitude d'usage des verbes falloir et devoir ( "nous devons... », " un vœu de vérité qui doit nous conduire... »). Ces verbes participent de la construction discursive du leadership (Bull et Fetzer 2012). 
17. On remarquera que personne sur la toile ne semble se souvenir des affaires ni du procès de Jacques Chirac (il a échappé à la prison du fait de sa maladie). Bien que le réseau constitue un lieu de discussion, il prouve le danger de l'oubli que $\mathrm{H}$. Arendt relève quand elle fait le rapprochement entre la mémoire et la profondeur de l'existence humaine (1972:125). Sans cet aspect, le dictat de la pensée au nom de la discussion publique risque de gommer les faits du passé en déformant la réalité. Ce qui donne des nouveaux modèles comme autorité à suivre.

\section{ABSTRACTS}

This article proposes an analysis of the reception of a political leader's authority on Twitter. The case study selected is the comments of the internet users to the French President Emmanuel Macron's New Year's Eve Message in 2018. The objective of this discursive and argumentative analysis is to investigate a contemporary phenomenon: the contestation of political figures' authority on the social networks, and more specifically on Twitter.

Cet article propose une étude de la réception de l'autorité d'un dirigeant politique sur Twitter. Le cas de figure analysé est celui des réactions à l'allocution de Nouvel An d'Emmanuel Macron en 2018. L'analyse discursive et argumentative des commentaires d'internautes a pour objectif d'explorer un phénomène contemporain : la mise en question de l'autorité du politique sur les réseaux sociaux, et plus particulièrement sur Twitter.

\section{INDEX}

Mots-clés: Autorité, Argument ad hominem, Argument ad personam, Macron (Emmanuel), Twitter

Keywords: Authority, Ad hominem, Ad personam, Macron (Emmanuel), Twitter

\section{AUTHOR}

\section{KEREN SADOUN-KERBER}

Université de Tel Aviv 